Check for updates

Cite this: J. Mater. Chem. B, 2018, 6, 7728

Received 4th September 2018, Accepted 10th October 2018

DOI: $10.1039 / c 8 t b 02325 e$

rsc.li/materials-b

\title{
Bifunctional scaffolds for the photothermal therapy of breast tumor cells and adipose tissue regeneration
}

\author{
Xiuhui Wang, (D) ab Jing Zhang, ${ }^{a b}$ Jingchao Li, (D) ${ }^{\text {ab }}$ Ying Chen, ${ }^{\text {ab }}$ Yazhou Chen, ${ }^{\text {ab }}$ \\ Naoki Kawazoe ${ }^{a}$ and Guoping Chen (D) *ab
}

\begin{abstract}
Breast cancer is a major public health issue, whose morbidity and mortality are increasing across the world. It is still a challenge to completely ablate breast tumor cells and reconstruct tumor-initiated breast defects after surgical resection. Porous scaffolds with hyperthermal and tissue regeneration functions are a desirable option to achieve these effects. In this study, bifunctional composite porous scaffolds of gold nanorods (AuNRs) and gelatin with well controlled pore structures were prepared by introducing AuNRs into the porous matrices of gelatin and using ice particulates as a porogen material. The AuNRs-gelatin composite scaffolds exhibited a high photothermal conversion effect, whose photothermal temperature could be modulated by the amount of incorporated AuNRs, NIR laser power intensity and irradiation time. The AuNRs-gelatin composite scaffolds exhibited an excellent photothermal ablation capacity toward breast tumor cells in vitro and in vivo. Furthermore, the AuNRs-gelatin porous scaffolds supported cell adhesion and promoted the proliferation and adipogenic differentiation of human bonemarrow derived mesenchymal stem cells (hMSCs). Consequently, the AuNRs-gelatin scaffolds could not only provide photothermal therapy for breast tumors but also promote the adipogenic differentiation of stem cells for adipose tissue regeneration.
\end{abstract}

\section{Introduction}

Breast cancer is one of the major threats to human health because of its increasing morbidity and mortality. ${ }^{1,2}$ To date, surgical resection, chemotherapy, radiotherapy and their combination have been widely used to treat breast tumors in the clinic. ${ }^{3}$ However, surgical resection cannot completely eliminate all the tumor cells and large breast defects always remain, which are difficult to self-heal. ${ }^{4}$ Chemotherapy and radiotherapy can give rise to adverse effects such as drugresistance and radio-resistance..$^{5-7}$ To date, it still remains a big challenge to realize breast reconstruction after tumor therapy. ${ }^{8}$ Therefore, it is necessary to design highly functional biomaterials that can be used to effectively ablate tumor cells and simultaneously promote breast reconstruction such as bioactive scaffolds. ${ }^{9,10}$

\footnotetext{
${ }^{a}$ Tissue Regeneration Materials Group, Research Center for Functional Materials, National Institute for Materials Science, 1-1 Namiki, Tsukuba, Ibaraki 305-0044, Japan. E-mail: Guoping.Chen@nims.go.jp; Fax: +81-29-860-4714; Tel: $+81-29-860-4496$

${ }^{b}$ Department of Materials Science and Engineering, Graduate School of Pure and Applied Sciences, University of Tsukuba, 1-1-1 Tennodai, Tsukuba,

Ibaraki 305-8571, Japan
}

In recent years, photothermal therapy (PTT), as a minimally invasive and highly efficient antitumor strategy, has attracted great attention. ${ }^{11}$ Various photothermal conversion agents such as carbon-based nanomaterials, copper sulfide nanoparticles and gold-based nanomaterials have been extensively explored. ${ }^{12-14}$ Among them, gold nanoparticles, especially gold nanorods (AuNRs), have received increasing attention due to their facile preparation, high photothermal conversion efficiency and good cytocompatibility. ${ }^{15}$ However, efficacious and site-specific delivery of free nanoparticles to the tumor site still has some problems. ${ }^{16}$ Upon intravenous injection, most of the free nanoparticles (NPs) undergo uptake by macrophages of the mononuclear phagocyte system, which results in high accumulation of NPs in healthy organs such as the liver and the spleen. ${ }^{17}$ To address these issues, immobilization of PTT NPs into three-dimensional (3D) scaffolds has been recently explored to realize repeat heating and local tumor therapy. ${ }^{18-21}$

Besides the photothermal therapy of breast tumor, adipose tissue regeneration is required to achieve breast reconstruction. ${ }^{22,23}$ It has been reported that adipose tissue can be regenerated by combining a 3D biodegradable porous scaffold with adiposederived stromal cells or bone-marrow derived mesenchymal stem cells. ${ }^{24,25}$ A 3D scaffold for adipose tissue engineering should bear soft tissue-like mechanical properties, biocompatibility 
and biodegradability. ${ }^{25}$ Gelatin has been used for the preparation of tissue regeneration scaffolds because of its high bioactivity, low antigenicity and low immunogenicity. ${ }^{26,27}$ Gelatin scaffolds have been reported to be favorable for adipose tissue engineering because of their soft tissue-like mechanical properties, as compared to stiff scaffolds such as the poly lactic-co-glycolic acid scaffold. ${ }^{25,28-30}$ In addition, 3D scaffolds should have an appropriate pore structure with open and interconnected pores to promote cell penetration and nutrition and metabolite exchange. ${ }^{31}$ Although a variety of methods have been explored to fabricate porous scaffolds such as electrospinning, 3D printing and porogen leaching methods, ${ }^{32-34}$ the freeze-drying method is one of the most commonly used methods for scaffold preparation of naturally derived polymers such as collagen and gelatin. ${ }^{35}$ Pre-prepared ice particulates can be mixed with naturally derived polymers before freezedrying to precisely control the pore structures of scaffolds. ${ }^{36,37}$

Based on the above considerations, in this study, bifunctional composite scaffolds of AuNRs and gelatin were prepared by incorporating AuNRs in gelatin matrices through a freezedrying method. Pre-prepared ice particulates were used as a porogen material and mixed with the AuNRs/gelatin mixture solution to control the pore structures of the composite scaffolds. The photothermal performance of the composite scaffolds under near infrared (NIR) laser irradiation at various power intensities was investigated. Their photothermal killing efficiency of breast tumor cells was evaluated by in vitro cell culture and in vivo animal experiments. Furthermore, the composite scaffolds were used for the $3 \mathrm{D}$ culture of human bone-marrow derived mesenchymal stem cells (hMSCs) to explore their capacity to promote the adipogenic differentiation of hMSCs.

\section{Materials and methods}

\section{Synthesis and characterization of gelatin-coated AuNRs}

AuNRs were synthesized by a seed-mediated growth method. ${ }^{38,39}$ Firstly, Au seed solution was prepared via the reduction of $\mathrm{HAuCl}_{4}$ by adding $\mathrm{NaBH}_{4}$ solution $\left(0.01 \mathrm{~mol} \mathrm{~L}{ }^{-1}, 1.2 \mathrm{~mL}\right)$ into a mixture of hexadecyltrimethylammonium bromide (CTAB, $0.1 \mathrm{~mol} \mathrm{~L}^{-1}$, $15.0 \mathrm{~mL})$ and $\mathrm{HAuCl}_{4}\left(0.01 \mathrm{~mol} \mathrm{~L}{ }^{-1}, 0.5 \mathrm{~mL}\right)$ solutions. The prepared $\mathrm{Au}$ seeds were used within 2 hours. Subsequently, a growth solution was obtained by mixing CTAB $\left(0.1 \mathrm{~mol} \mathrm{~L}^{-1}\right.$, $100.0 \mathrm{~mL})$ solution with $\mathrm{HAuCl}_{4}\left(0.01 \mathrm{~mol} \mathrm{~L}{ }^{-1}, 5.0 \mathrm{~mL}\right), \mathrm{HCl}$ $\left(1 \mathrm{~mol} \mathrm{~L}{ }^{-1}, 2.0 \mathrm{~mL}\right), \mathrm{AgNO}_{3}\left(0.01 \mathrm{~mol} \mathrm{~L}^{-1}, 1.1 \mathrm{~mL}\right)$ and ascorbic acid $\left(0.1 \mathrm{~mol} \mathrm{~L}^{-1}, 0.8 \mathrm{~mL}\right)$ solutions in sequence. Finally, $0.24 \mathrm{~mL}$ of Au seed solution was added into the growth solution under gentle stirring and then left undisturbed for 12 hours to generate the AuNRs. The AuNRs were collected by centrifugation and washed with ultrapure water to remove the residual CTAB.

In order to avoid agglomeration of AuNRs and to make a homogeneously distributed AuNR suspension in ultrapure water, the AuNRs were coated with gelatin by dispersing them into $0.5 \%(\mathrm{w} / \mathrm{v})$ gelatin solution under stirring for 24 hours. The gelatin-coated AuNRs were obtained by centrifugation.
The gelatin-coated AuNRs were resuspended in ultrapure water for the following experiments.

The morphology of the gelatin-coated AuNRs was characterized using transmission electron microscopy (TEM, JEOL 2011F, Japan). The size of the AuNRs was measured from TEM images using Image J software (Image J2, NIH). The visible-near infrared (Vis-NIR) spectrum of the aqueous solution of the AuNRs was measured using a UV-660 UV-Vis spectrophotometer (Jasco Corp., Japan).

\section{Preparation and characterization of AuNRs-gelatin composite scaffolds}

AuNRs-gelatin composite porous scaffolds were prepared by freeze-drying the mixture solution of the gelatin-coated AuNRs, gelatin and pre-prepared ice particulates as previously reported. ${ }^{21,36,40}$ Briefly, ice particulates were first prepared by spraying ultrapure water into liquid nitrogen and sieving with two sieves having respective mesh sizes of $425 \mu \mathrm{m}$ and $500 \mu \mathrm{m}$ to obtain ice particulates having a size range of $425-500 \mu \mathrm{m}$. A $70 \%$ acetic acid solution of $8(\mathrm{w} / \mathrm{v}) \%$ gelatin was mixed with a 4.0 or $8.0 \mathrm{mM}$ AuNR solution at a ratio of $1: 1(\mathrm{v} / \mathrm{v})$ under sonication to prepare the AuNRs/gelatin mixture solutions. The final concentration of gelatin in the mixture solutions was $4(\mathrm{w} / \mathrm{v}) \%$ while the AuNR concentration was 2.0 and $4.0 \mathrm{mM}$ because our previous study showed that these two concentrations had a good heating effect. ${ }^{21}$ The ice particulates, gelatin aqueous solution and AuNRs/gelatin mixture solution were kept in a chamber at $-4{ }^{\circ} \mathrm{C}$ for 6 hours to balance the temperature. Subsequently, the ice particulates were homogenously mixed with the AuNRs/gelatin mixture solution at a ratio of $7: 3(\mathrm{w} / \mathrm{v})$. The mixture of ice particulates and AuNRs-gelatin solution was poured into a silicone mold. Finally, the whole constructs were frozen at $-20{ }^{\circ} \mathrm{C}$ for 12 hours and transferred to a $-80{ }^{\circ} \mathrm{C}$ freezer for 4 hours. The frozen constructs were freeze-dried in a freeze dryer (FDU-2200, Japan) for 3 days and cross-linked by 1-ethyl-3-(3-dimethylaminopropyl) carbodiimide and $\mathrm{N}$-hydroxysuccimide (EDC/NHS) to obtain the AuNRs-gelatin composite porous scaffolds. The morphology of the AuNRs-gelatin composite scaffolds was characterized using field emission scanning electron microscopy (FESEM: SU8220, Hitachi, Japan). The AuNRs-gelatin composite scaffold prepared with a AuNR concentration of 2.0 and $4.0 \mathrm{mM}$ was defined as $2.0 \mathrm{mM}$ AuNRs-gelatin scaffold and $4.0 \mathrm{mM}$ AuNRs-gelatin scaffold, respectively. A gelatin scaffold without the incorporation of AuNRs was also prepared as a control with the above-mentioned procedures without addition of the AuNRs.

The pore size of the gelatin scaffold and AuNRs-gelatin composite scaffolds was analyzed by measuring the diameters of pores from the four SEM images of each type of scaffold using ImageJ software. The photothermal performance of the gelatin scaffold, $2.0 \mathrm{mM}$ AuNRs-gelatin scaffold and $4.0 \mathrm{mM}$ AuNRs-gelatin scaffold was investigated under irradiation with an $805 \mathrm{~nm}$ laser (Thorlabs Inc., USA). The AuNRs-gelatin composite scaffolds were cut into cubes with a dimension of $5.0 \times 3.0 \times 1.0 \mathrm{~mm}$. The samples were hydrated with culture medium and then exposed to a near infrared (NIR) laser 
(805 nm). The temperature change of the AuNRs-gelatin composite scaffolds under NIR laser irradiation with different power intensities $\left(1.3,1.4,1.5\right.$ or $\left.1.6 \mathrm{~W} \mathrm{~cm}^{-2}\right)$ was recorded using a digital thermometer (As one Corp., Osaka, Japan) in real time.

\section{Photothermal ablation of in vitro cultured breast tumor cells in the AuNRs-gelatin scaffolds}

The AuNRs-gelatin composite porous scaffolds $(5.0 \times 3.0 \times$ $1.0 \mathrm{~mm}$ ) were sterilized with $70 \%$ ethanol aqueous solution. Human breast tumor cells that stably express luciferase (MDA-MB231-Luc) were obtained from the Japanese collection of research bioresources cell bank (Osaka, Japan). MDA-MB231Luc cells were cultured in L-15 medium supplemented with $15 \%$ FBS and L-glutamine $(2 \mathrm{mM})$ in a humidified incubator $\left(5 \% \mathrm{CO}_{2}, 37{ }^{\circ} \mathrm{C}\right)$. The subcultured breast tumor cells were harvested by treatment with a $0.05 \%$ trypsin-EDTA solution. The harvested MDA-MB231-Luc cells were resuspended in L-15 medium to prepare a cell suspension solution at a concentration of $1.0 \times 10^{7}$ cells per $\mathrm{mL}$ for cell seeding. Subsequently, $15 \mu \mathrm{L}$ of the cell suspension solution was dropped on the top side of the scaffold cubes. After being cultured for 3 hours, the scaffold cubes were turned upside down and another $15 \mu \mathrm{L}$ of cell suspension solution was dropped on the other side of the scaffolds. The cell/scaffold constructs were transferred to a 24-well culture plate with $1 \mathrm{~mL}$ of L-15 medium in each well.

After being cultured for 24 hours, the cell/scaffold constructs were irradiated with NIR laser $(805 \mathrm{~nm})$ at different power intensities ( 1.3 or $1.6 \mathrm{~W} \mathrm{~cm}^{-2}$ ). After laser irradiation, the cell/scaffold constructs were cultured for another 5 hours for live/dead cell staining. The calcein-AM/PI double staining kit (Dojindo, Japan) was used to stain live and dead cells in the composite scaffolds before and after laser irradiation. The stained samples were observed using an inverted fluorescence microscope (Olympus, Japan). In addition, cell viability in the scaffolds before and after irradiation with a power intensity of 1.3 or $1.6 \mathrm{~W} \mathrm{~cm}^{-2}$ for 3, 5, 6 and 8 minutes was quantified by WST-1 assay. $400 \mu \mathrm{L}$ of WST-1 reagent diluted with medium (1:10) was added into each well containing the cell/scaffold constructs and the cells were cultured for another 3 hours. Then, the absorbance of the WST-1 solution at $440 \mathrm{~nm}$ was measured using a microplate reader (Benchmark Plus, Bio-Rad, Hercules, CA, USA). Three samples were used for each measurement to calculate the average and standard deviation.

\section{Photothermal ablation of in vivo implanted breast tumor cells by AuNRs-gelatin scaffolds}

The MDA-MB231-Luc cells were seeded in the cubes $(5.0 \times 3.0 \times$ $1.0 \mathrm{~mm}$ ) of the gelatin porous scaffold and $2.0 \mathrm{mM}$ AuNRs-gelatin scaffold and cultured in vitro for 3 days. The tumor cells/scaffold constructs were subcutaneously implanted in the back of 6-weekold female athymic nude mice. The nude mice were divided into four groups: tumor cells/gelatin scaffold, tumor cells/AuNRsgelatin scaffold, tumor cells/gelatin scaffold + NIR laser irradiation, tumor cells/AuNRs-gelatin scaffold + NIR laser irradiation. After implantation for 6 days, the implantation sites of the mice were irradiated with NIR laser $(805 \mathrm{~nm})$ at a power intensity of $1.3 \mathrm{~W} \mathrm{~cm}^{-2}$ for 10 minutes and $1.6 \mathrm{~W} \mathrm{~cm}^{-2}$ for 8 minutes. After 1 day, a whole-body bioluminescence image was taken with an in vivo vision system (IVIS Lumina II, Japan) to evaluate the in vivo photothermal ablation effect of the $2.0 \mathrm{mM}$ AuNRsgelatin scaffold. All the animal experiment procedures were approved by the Animal Experiments Committee of the National Institute for Materials Science and the experiment was conducted according to the committee guidelines.

\section{Adipogenic differentiation of hMSCs in AuNRs-gelatin composite scaffolds}

The hMSCs at passage 2 (P2) were purchased from Lonza (Walkersville MD, USA) and subcultured in MSCBM medium (Lonza, Swiss). The subcultured hMSCs at $\mathrm{P} 4$ were harvested by treatment with a trypsin/EDTA solution and resuspended in DMEM medium to prepare the cell suspension solution at a cell concentration of $4.0 \times 10^{6}$ cells per mL for cell seeding. $15 \mu \mathrm{L}$ of cell suspension solution was dropped on the top side of the gelatin scaffold, $2.0 \mathrm{mM}$ AuNRs-gelatin scaffold and $4.0 \mathrm{mM}$ AuNRs-gelatin scaffold. After being cultured for 3 hours, the scaffold cubes were turned upside down and another $15 \mu \mathrm{L}$ of cell suspension solution was dropped on the other side of the scaffolds. DMEM medium was changed every 3 days with the addition of fresh $1 \mu \mathrm{M}$ dexamethasone, $0.5 \mathrm{mM}$ methyl-isobutylxanthine, $100 \mu \mathrm{M}$ indomethacin and $10 \mu \mathrm{g} \mathrm{mL}{ }^{-1}$ insulin.

After being cultured for 1 day, cell attachment was analyzed by SEM observation. After 1 day culture, the hMSCs/scaffold constructs were washed with PBS solution, fixed with $2.5 \%$ glutaraldehyde, washed with water and freeze-dried. The freezedried hMSCs/scaffold constructs were observed by FE-SEM to study cell adhesion and morphology in the scaffolds.

Cell proliferation was investigated by measuring the amount of DNA after being cultured for 1, 7 and 14 days. The hMSCs/ scaffold constructs were washed with PBS 3 times, freeze-dried and digested with $400 \mu \mathrm{g} \mathrm{mL}^{-1}$ papain solution containing $5 \mathrm{mM}$ EDTA and $5 \mathrm{mM}$ L-cysteine in $0.1 \mathrm{M}$ phosphate buffer $(\mathrm{pH}$ 6.0). An aliquot of papain digest solution was used to measure the amount of DNA with Hoechst 33258 dye (SigmaAldrich, USA) using a fluorescence spectrometer (FP8500, JASCO, Japan). Three samples were used for each measurement to calculate the average and standard deviation.

Adipogenic differentiation of the hMSCs in the AuNRsgelatin composite scaffolds prepared with different AuNR concentrations (0, 2.0 and $4.0 \mathrm{mM}$ ) was analyzed by using Oil Red $\mathrm{O}$ staining and expression of adipogenesis genes. Oil Red $\mathrm{O}$ staining was carried out after 14 days of culture. The hMSCs/ scaffold constructs were washed with PBS, fixed with $4 \%$ paraformaldehyde and washed with water. Then, the samples were soaked in $60 \%$ isopropanol for 5 minutes and subsequently soaked in Oil Red $\mathrm{O}$ working solution for 5 minutes. After being washed with water, the stained constructs were observed using an optical microscope. In addition, the stained samples were dried in air and the Oil Red O dye was extracted by treatment with isopropanol for 2 hours at room temperature. The absorbance of the extracted Oil Red $\mathrm{O}$ dye was measured 
Table 1 The primers and probes for real-time PCR

\begin{tabular}{ll}
\hline mRNA & Oligonucleotide \\
\hline GAPDH & Hs99999905_m1 \\
PPARG & Hs01115510_m1 \\
LPL & Hs00173425_m1 \\
FABP4 & Hs00609791_m1 \\
FASN & Hs00188012_m1 \\
CEBPA & Hs00269972_s1
\end{tabular}

using a plate reader at a wavelength of $540 \mathrm{~nm} .{ }^{41}$ The expression of adipogenesis genes including CCAAT/enhancer binding protein (CEBPA), peroxisome proliferator-activated receptor gamma (PPARG), lipoprotein lipase (LPL), fatty acid binding protein 4 (FABP4) and fatty acid synthase (FASN) in the hMSCs/ scaffold constructs was analyzed by real time PCR (RT-PCR). After being cultured for 14 days, the samples were washed with PBS 3 times and frozen in liquid nitrogen. The frozen samples were crushed into a powder using an electric crusher and dissolved in Sepasol solution (Nacalai Tesque, Japan) to isolate RNA. The RNA was converted to cDNA by a first strand cDNA synthesis kit (Applied Biosystems, USA). The real-time polymerase chain reaction (PCR) was performed using a 7500 Real-Time PCR system (Applied Biosystems, USA). ${ }^{42}$ The primers and probes are listed in Table 1. The expression of GAPDH was used as an endogenous control. Relative gene expression was calculated using a $2^{-\Delta \Delta C t}$ method. The hMSCs at P4 used for cell seeding were used as a control for comparison. Three samples were used for each measurement to calculate the average and standard deviation.

\section{Statistical analysis}

All quantitative experiments were repeated in triplicate $(n=3)$ and the results are expressed as mean \pm standard deviation. The statistical analysis of experimental data was performed by using one-way analysis of variance statistical analysis (ANOVA). A $p$ value of 0.05 was set as the level of significance and the data were classified according to their $p$ values and denoted by $\left(^{*}\right)$ for $p<0.05,\left(^{* *}\right)$ for $p<0.01$, and $\left({ }^{* *}\right)$ for $p<0.001$.

\section{Results}

\section{Characterization of the AuNRs}

The gross appearance of the colloidal solution of AuNRs (Fig. 1a) showed that the gelatin-coated gold nanoparticles were homogeneously dispersed in pure water without aggregation. TEM images (Fig. 1b and c) indicated that the gold nanoparticles were uniformly distributed without aggregation and had a rod-like shape with dimensions of $66.1 \pm 2.3 \mathrm{~nm} \times 13.6 \pm 2.5 \mathrm{~nm}$. The visible-near infrared (Vis-NIR) absorption spectrum (Fig. 1d) showed that the AuNRs had a strong absorption peak in the near infrared region, which is beneficial for photothermal therapy.

\section{Characterization of the gelatin scaffold and AuNRs-gelatin composite scaffolds}

SEM observation showed that the AuNRs-gelatin composite scaffolds possessed spherical large micropores with good

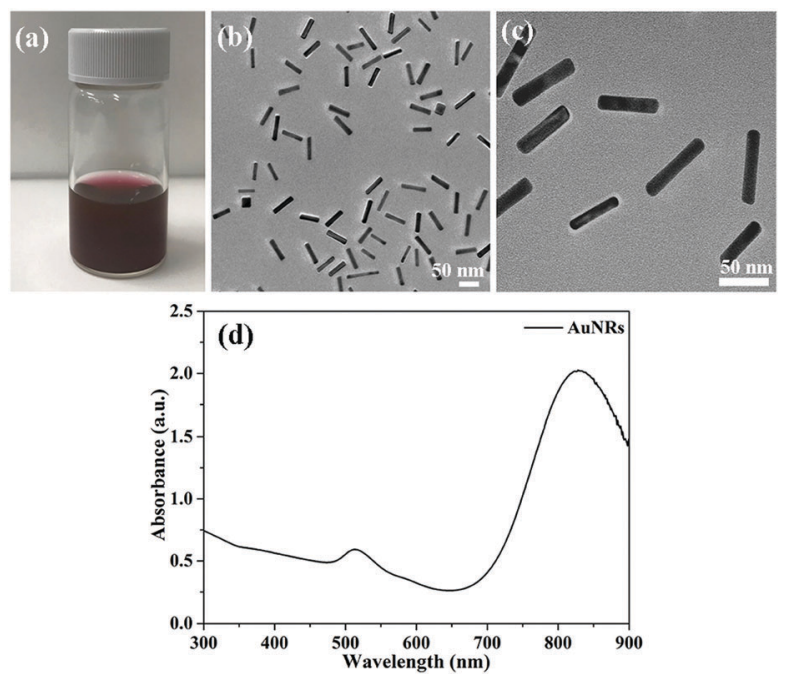

Fig. 1 Gross appearance of the colloidal solution of the gelatin-coated AuNRs in pure water (a), TEM images of the AuNRs at low (b) and high (c) magnifications and VIS-NIR spectrum of AuNR colloidal solution (d).

interconnectivity, which were the same as those of the gelatin porous scaffold (Fig. 2a-c). The size of the spherical large micropores in the gelatin scaffold, $2.0 \mathrm{mM}$ AuNRs-gelatin scaffold and $4.0 \mathrm{mM}$ AuNRs-gelatin scaffold was $439 \pm 38 \mu \mathrm{m}$, $442 \pm 21 \mu \mathrm{m}$ and $435 \pm 44 \mu \mathrm{m}$, respectively. The large micropore size was in the same range because the same ice particulates having a diameter of $425-500 \mu \mathrm{m}$ were used for preparation of all three types of scaffolds. Small micropores connecting the large micropores were observed on the walls of the spherical large micropores. The size of the small micropores in the gelatin scaffold, $2.0 \mathrm{mM}$ AuNRs-gelatin scaffold and $4.0 \mathrm{mM}$ AuNRs-gelatin scaffold was $66 \pm 25 \mu \mathrm{m}$, $76 \pm 18 \mu \mathrm{m}$ and $69 \pm 23 \mu \mathrm{m}$, respectively. The three types of scaffolds had the same range of small micropores. Observation at a high magnification showed that the AuNRs were individually distributed on the micropore wall of the composite scaffolds (Fig. 2e and f). No AuNRs were observed in the gelatin scaffold (Fig. 2d).

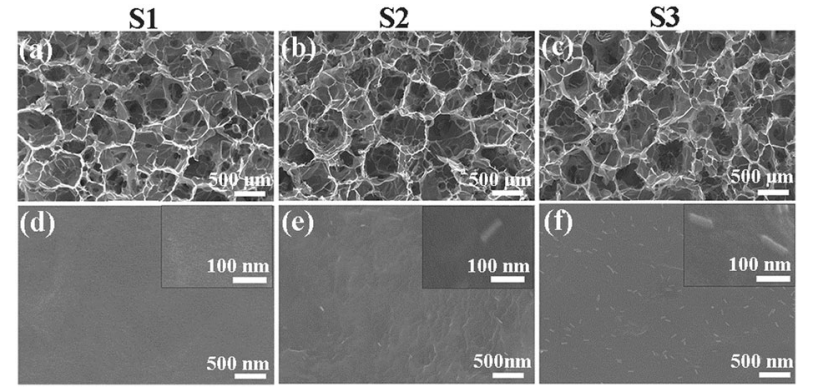

Fig. 2 SEM images of the gelatin scaffold (a and d), $2.0 \mathrm{mM}$ AuNRsgelatin scaffold ( $b$ and e) and $4.0 \mathrm{mM}$ AuNRs-gelatin scaffold ( $c$ and f) at a low magnification $(a-c)$ and a high magnification (d-f). S1, S2 and S3 indicate gelatin scaffold, $2.0 \mathrm{mM}$ AuNRs-gelatin scaffold and $4.0 \mathrm{mM}$ AuNRs-gelatin scaffold, respectively. The insets show the magnified images of the corresponding scaffolds. 


\section{Photothermal performance of the AuNRs-gelatin composite scaffolds}

The temperature of the AuNRs-gelatin scaffolds increased when the scaffolds were irradiated with NIR laser of $805 \mathrm{~nm}$ (Fig. 3). The photothermal temperature of the AuNRs-gelatin scaffolds could be effectively modulated by changing the laser power intensity and altering the amount of AuNRs. The AuNRsgelatin scaffolds showed different photothermal efficiency under a variable laser power intensity (Fig. 3a and b). A higher laser power intensity resulted in a higher temperature. The temperature change data (Table 2) indicated that the temperature change of the gelatin scaffold, 2.0 mM AuNRs/ gelatin scaffold and $4.0 \mathrm{mM}$ AuNRs/gelatin scaffold was $4.4 \pm$ 0.9-8.9 $\pm 0.7{ }^{\circ} \mathrm{C}, 23.2 \pm 0.8-32.9 \pm 0.9{ }^{\circ} \mathrm{C}$ and $28.1 \pm 0.9-41.0 \pm$ $1.0{ }^{\circ} \mathrm{C}$, respectively, when the laser power intensity varied from $1.3 \mathrm{~W} \mathrm{~cm} \mathrm{~cm}^{-2}$ to $1.6 \mathrm{~W} \mathrm{~cm} \mathrm{~cm}^{-2}$. When different scaffolds were compared, the temperature change of the gelatin scaffold under NIR laser irradiation was very slow while that of the AuNRs-gelatin scaffolds increased rapidly (Fig. 3c and d). The temperature of the $4.0 \mathrm{mM}$ AuNRs-gelatin scaffolds was higher than that of the $2.0 \mathrm{mM}$ AuNRs-gelatin scaffolds under the same power density, which indicated that the increased amount of incorporated AuNRs enhanced the photothermal conversion efficiency of the composite scaffolds.

\section{Photothermal ablation of in vitro cultured breast tumor cells by the AuNRs-gelatin composite scaffolds}

The photothermal ablation of breast tumor cells cultured in the AuNRs-gelatin scaffolds was explored by NIR laser irradiation with a power intensity of 1.3 and $1.6 \mathrm{~W} \mathrm{~cm}^{-2}$. Live/dead staining showed that almost all the tumor cells were live within the porous scaffolds before NIR laser irradiation (Fig. 4a-c).
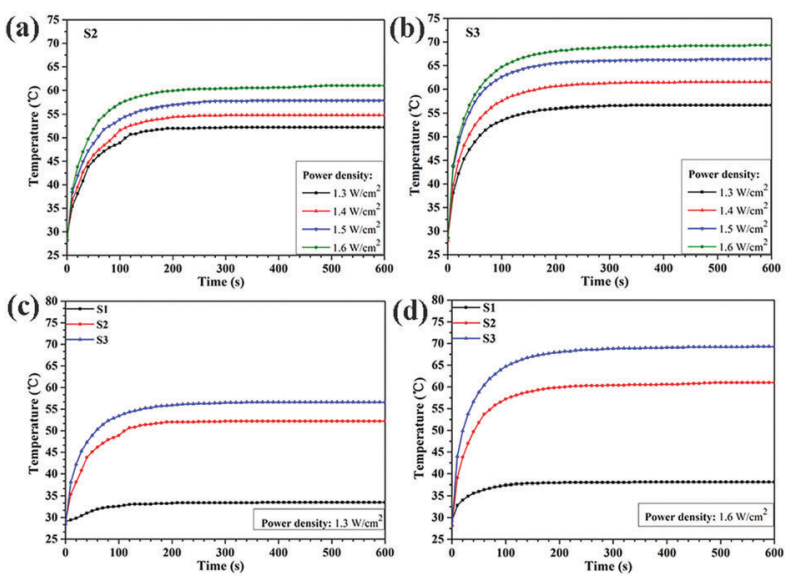

Fig. 3 Heating curves of the AuNRs-gelatin composite scaffolds under continuous irradiation with $805 \mathrm{~nm}$ laser. Temperature-irradiation time curves of the $2.0 \mathrm{mM}$ AuNRs-gelatin scaffold (a) and $4.0 \mathrm{mM}$ AuNRsgelatin scaffold (b) under NIR laser irradiation at a power intensity of 1.3, $1.4,1.5$ and $1.6 \mathrm{~W} \mathrm{~cm}^{-2}$. Temperature-irradiation time curves of the gelatin scaffold, $2.0 \mathrm{mM}$ AuNRs-gelatin scaffold and $4.0 \mathrm{mM}$ AuNRs-gelatin scaffold under NIR laser irradiation at a power intensity of $1.3 \mathrm{~W} \mathrm{~cm}^{-2}$ (c) and $1.6 \mathrm{~W} \mathrm{~cm}^{-2}$ (d). S1, S2 and S3 indicate gelatin scaffold, $2.0 \mathrm{mM}$ AuNRsgelatin scaffold and $4.0 \mathrm{mM}$ AuNRs-gelatin scaffold, respectively.
Table 2 Temperature change $\left({ }^{\circ} \mathrm{C}\right.$ ) of the gelatin scaffold (S1), $2.0 \mathrm{mM}$ AuNRs-gelatin scaffold (S2) and 4.0 mM AuNRs-gelatin scaffold (S3) after irradiation at different laser power densities for 10 minutes

\begin{tabular}{llll}
\hline & \multicolumn{2}{l}{ Sample } & \\
\cline { 2 - 4 } Power density & $\mathrm{S} 1$ & $\mathrm{~S} 2$ & $\mathrm{~S} 3$ \\
\hline $1.3 \mathrm{~W} \mathrm{~cm}^{-2}$ & $4.4 \pm 0.9$ & $23.2 \pm 0.8$ & $28.1 \pm 0.9$ \\
$1.4 \mathrm{~W} \mathrm{~cm}^{-2}$ & $6.9 \pm 0.6$ & $26.6 \pm 1.1$ & $33.5 \pm 1.3$ \\
$1.5 \mathrm{~W} \mathrm{~cm}^{-2}$ & $7.9 \pm 0.5$ & $29.6 \pm 0.7$ & $38.0 \pm 1.4$ \\
$1.6 \mathrm{~W} \mathrm{~cm}^{-2}$ & $8.9 \pm 0.7$ & $32.9 \pm 0.9$ & $41.0 \pm 1.0$
\end{tabular}

After NIR laser irradiation at a laser power intensity of $1.3 \mathrm{~W} \mathrm{~cm}^{-2}$ for 8 minutes and a laser power intensity of $1.6 \mathrm{~W} \mathrm{~cm}^{-2}$ for 6 minutes, almost all the breast tumor cells were dead in the AuNRs-gelatin composite scaffolds, while the breast tumor cells still remained alive in the gelatin scaffolds (Fig. 4d-i). The quantification of cell viability showed that the cellular viability of the MDA-MB231-Luc cells cultured in the gelatin scaffold experienced no significant change before and after NIR laser irradiation, while cellular viability in the AuNRs-gelatin composite scaffolds significantly decreased after NIR laser irradiation (Fig. $4 \mathrm{j}$ and $\mathrm{k}$ ). The increase in irradiation time significantly decreased cell viability. The cell viability of the MDA-MB231-Luc cells cultured in the $2.0 \mathrm{mM}$ AuNRs-gelatin scaffold and 4.0 mM AuNRs-gelatin scaffold decreased to 0\% after NIR laser irradiation at a power intensity of $1.3 \mathrm{~W} \mathrm{~cm}^{-2}$ for

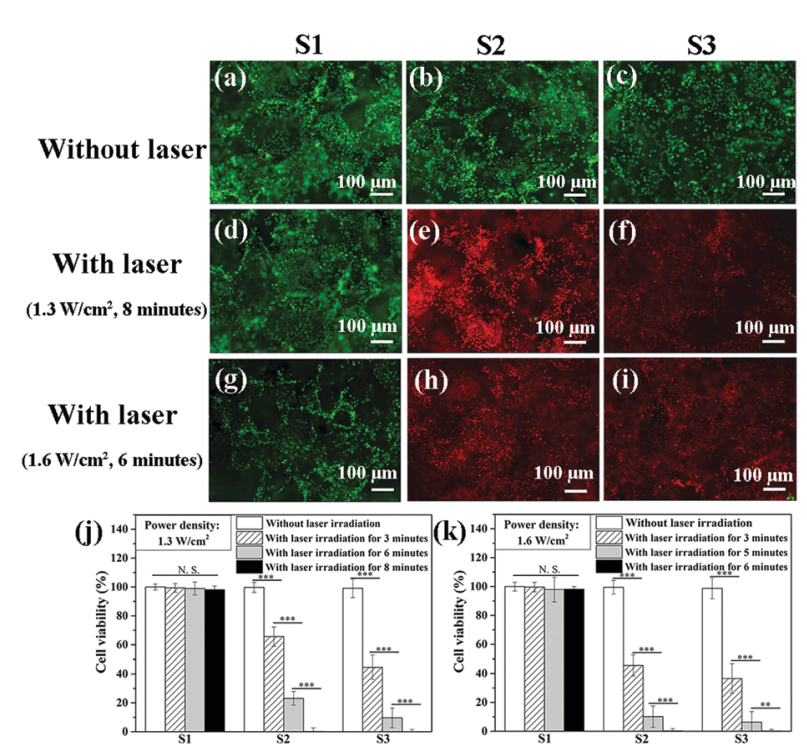

Fig. 4 Live/dead staining of breast tumor cells (MDA-MB231-Luc cells) cultured in the gelatin scaffold ( $a, d$ and g), $2.0 \mathrm{mM}$ AuNRs-gelatin scaffold (b, e and $h$ ) and $4.0 \mathrm{mM}$ AuNRs-gelatin scaffold (c, f and i) without (a-c) and with NIR laser irradiation (d-i) at a laser power intensity of $1.3 \mathrm{~W} \mathrm{~cm}^{-2}$ for 8 minutes $(d-f)$ and a laser power intensity of $1.6 \mathrm{~W} \mathrm{~cm}^{-2}$ for 6 minutes $(\mathrm{g}-\mathrm{i})$. Green color indicates live cells stained by calcein-AM while red color indicates dead cells stained by PI. Viability of the MDA-MB231-Luc cells cultured in the gelatin scaffold and AuNRs-gelatin composite scaffolds after irradiation for different times with a laser power intensity of $1.3 \mathrm{~W} \mathrm{~cm}^{-2}$ (j) and $1.6 \mathrm{~W} \mathrm{~cm}^{-2}$ (k). S1, S2 and S3 indicate gelatin scaffold, $2.0 \mathrm{mM}$ AuNRs-gelatin scaffold and $4.0 \mathrm{mM}$ AuNRs-gelatin scaffold, respectively. The data are presented as mean \pm standard deviation, $n=3$. No significant difference: N.S.; significant difference: ${ }^{*} p<0.05 ;{ }^{* *} p<0.01 ;{ }^{* \star *} p<0.001$. 
8 minutes or $1.6 \mathrm{~W} \mathrm{~cm}^{-2}$ for 6 minutes. The increase in NIR laser density resulted in a further decrease of cell viability. Under the same NIR laser irradiation conditions, the cells cultured in the $4.0 \mathrm{mM}$ AuNRs-gelatin scaffold exhibited a quicker decrease of viability than those cultured in the $2.0 \mathrm{mM}$ AuNRs-gelatin scaffold, suggesting that the increased amount of AuNRs raised the photothermal killing effect of the tumor cells.

\section{In vivo photothermal ablation of tumor cells by the AuNRs-gelatin composite scaffolds}

The in vivo photothermal ablation effect of the breast tumor cells by the AuNRs-gelatin scaffolds was evaluated by whole-body bioluminescence imaging after laser irradiation. MDA-MB-231-Luc cells were cultured in the gelatin scaffold and $2.0 \mathrm{mM}$ AuNRsgelatin scaffold. The cell/scaffold constructs were subcutaneously implanted on the backs of nude mice. After 6 days implantation, the implantation sites of the mice were irradiated with NIR laser. Bioluminescence images showed that the bioluminescent signal of the living MDA-MB231-Luc cells in the $2.0 \mathrm{mM}$ AuNRsgelatin scaffold evidently decreased after NIR laser irradiation, while that in the gelatin scaffold remained almost unchanged (Fig. 5). Irradiation at a laser power intensity of $1.6 \mathrm{~W} \mathrm{~cm}^{-2}$ for 8 minutes showed almost the same effect as that of irradiation at a laser power intensity of $1.3 \mathrm{~W} \mathrm{~cm}^{-2}$ for 10 minutes. The results indicated that most of the breast cancer cells were killed by the $2.0 \mathrm{mM}$ AuNRs-gelatin scaffold under NIR laser irradiation. The gross appearance of the mice showed that the skin tissue near the implanted $2.0 \mathrm{mM}$ AuNRs-gelatin scaffold was obviously damaged after NIR laser irradiation at a high power intensity $\left(1.6 \mathrm{~W} \mathrm{~cm}^{-2}\right)$ for 8 minutes (Fig. 6). Skin damage was suppressed when the
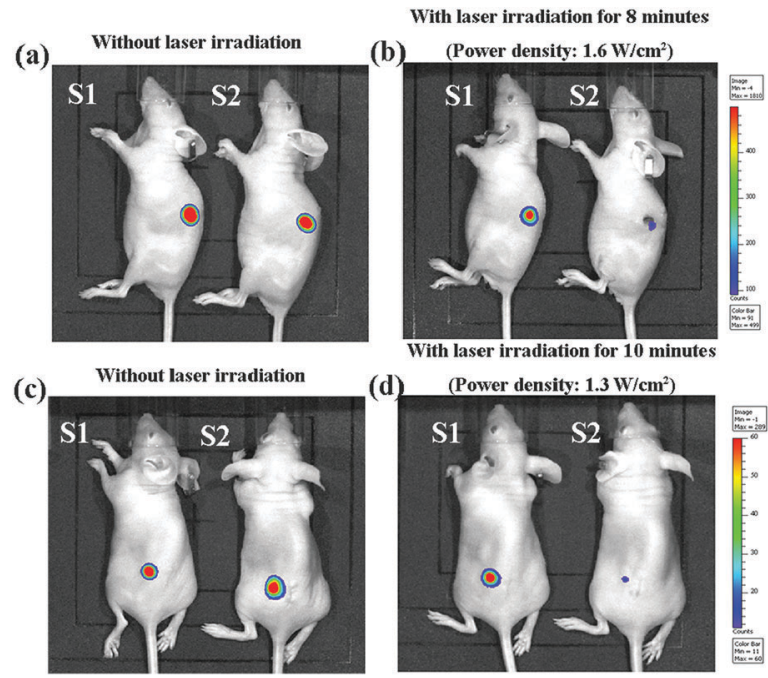

Fig. 5 Whole-body bioluminescence imaging of mice subcutaneously implanted with MDA-MB231-Luc cells/gelatin scaffold and MDA-MB231Luc cells/2.0 mM AuNRs-gelatin scaffold constructs before irradiation (a and c) and after NIR laser irradiation at a laser density of $1.6 \mathrm{~W} \mathrm{~cm}^{-2}$ for 8 minutes (b) and a laser density of $1.3 \mathrm{~W} \mathrm{~cm}^{-2}$ for 10 minutes (d). S1 and S2 indicate gelatin scaffold and $2.0 \mathrm{mM}$ AuNRs-gelatin scaffold, respectively. (a)

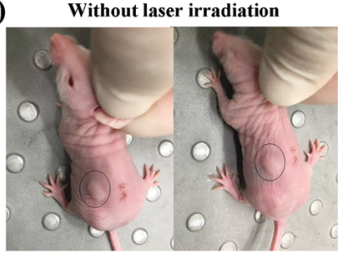

(c)

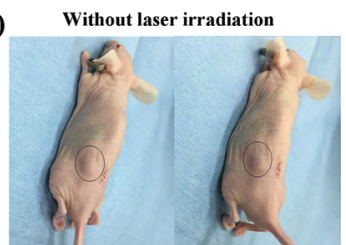

(b)

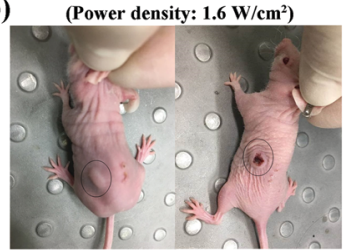

(d) With laser irradiation for 10 minutes
(Power density: 1.3 W/ $/ \mathrm{cm}^{2}$ )
With laser irradiation for 8 minutes

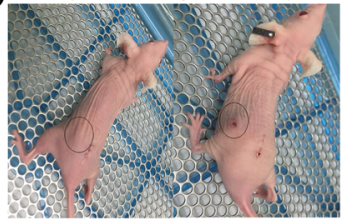

Fig. 6 Photographs of mice subcutaneously implanted with the MDAMB231-Luc cells/gelatin scaffold and MDA-MB231-Luc cells/2.0 mM AuNRs-gelatin scaffold constructs before irradiation (a and c) and after NIR laser irradiation at a laser density of $1.6 \mathrm{~W} \mathrm{~cm}^{-2}$ for 8 minutes (b) and a laser density of $1.3 \mathrm{~W} \mathrm{~cm}^{-2}$ for 10 minutes (d).

irradiation was conducted at a laser power intensity of $1.3 \mathrm{~W} \mathrm{~cm}^{-2}$ for 10 minutes.

\section{Adhesion and proliferation of hMSCs in the AuNRs-gelatin composite scaffolds}

To investigate the capacity of the AuNRs-gelatin composite scaffolds for adipose tissue regeneration, the composite scaffolds were used for the 3D culture of hMSCs. After 1 day culture, cell adhesion and distribution in the scaffolds were observed by SEM (Fig. 7). The SEM images showed that the hMSCs adhered well with typical filopodia on the wall of the micropores in all the gelatin and AuNRs-gelatin composite scaffolds. The cells were distributed throughout the scaffolds. DNA quantification (Fig. 8) showed that the DNA content increased significantly after being cultured for 1, 7 and 14 days in all the scaffolds. DNA content showed no significant difference among all the three types of scaffolds. The results indicate that all three types of scaffolds supported cell adhesion and promoted the proliferation of hMSCs. The incorporation of AuNRs into the gelatin scaffold had no significant influence on the adhesion and proliferation of hMSCs.

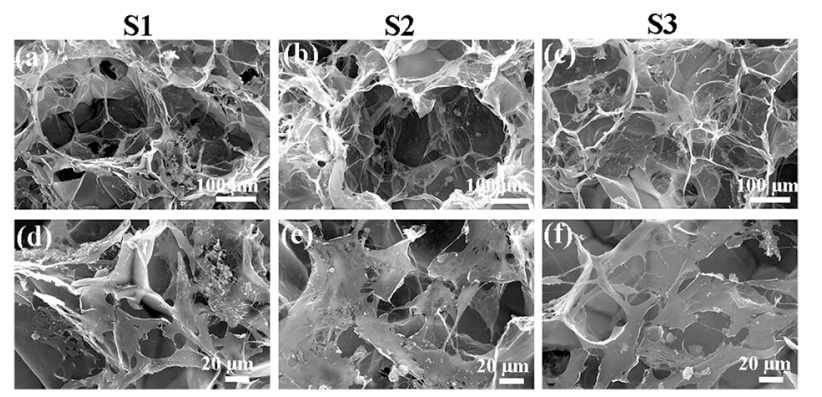

Fig. 7 SEM images of hMSCs/scaffold constructs at a low magnification $(a-c)$ and a high magnification $(d-f)$ after hMSCs were cultured in the gelatin scaffold (S1, a and d), $2.0 \mathrm{mM}$ AuNRs-gelatin scaffold (S2, b and e) and $4.0 \mathrm{mM}$ AuNRs-gelatin scaffold (S3, c and f) for 1 day. 


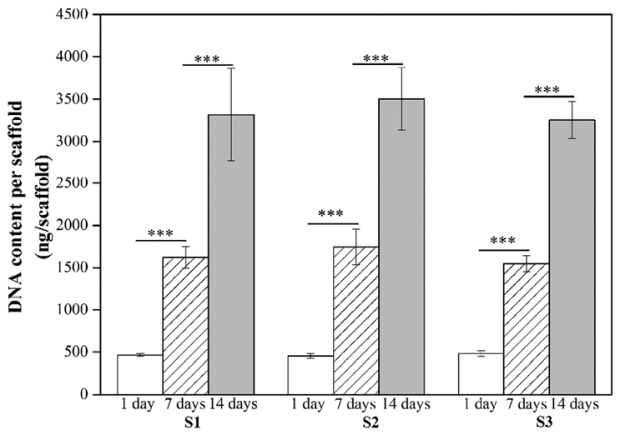

Fig. 8 Quantification of the DNA content of hMSCs/scaffold constructs after the hMSCs were cultured in the gelatin scaffold (S1), $2.0 \mathrm{mM}$ AuNRsgelatin scaffold (S2) and $4.0 \mathrm{mM}$ AuNRs-gelatin scaffold (S3) for 1, 7 and 14 days. The data are presented as mean \pm standard deviation, $n=3$. Significant difference: ${ }^{* *} p<0.001$.

\section{Adipogenic differentiation of hMSCs in the AuNRs-gelatin composite scaffolds}

The adipogenic differentiation of hMSCs in the AuNRs-gelatin composite scaffolds was analyzed by Oil Red O staining and the expression of adipogenesis genes after culture in adipogenic induction medium for 14 days. Oil Red O staining showed that lipid vacuoles were detected when the hMSCs were cultured in the gelatin scaffold, 2.0 mM AuNRs-gelatin scaffold and $4.0 \mathrm{mM}$ AuNRs-gelatin scaffold (Fig. 9a-c). Quantification of the extracted Oil Red $\mathrm{O}$ dye showed that the amount of lipid vacuoles exhibited no significant difference among the three types of scaffolds (Fig. 9d). Furthermore, the expression of genes encoding CEBPA, PPARG, LPL, FABP4 and FASN showed that all these genes were upregulated when the hMSCs were cultured in the gelatin scaffold and AuNRs-gelatin composite scaffolds in comparison with the subcultured hMSCs (control) (Fig. 10). The expression level of these genes showed no significant difference among the gelatin scaffold, 2.0 mM AuNRs-gelatin scaffold and 4.0 mM AuNRsgelatin scaffold. Oil Red $\mathrm{O}$ staining and gene expression results
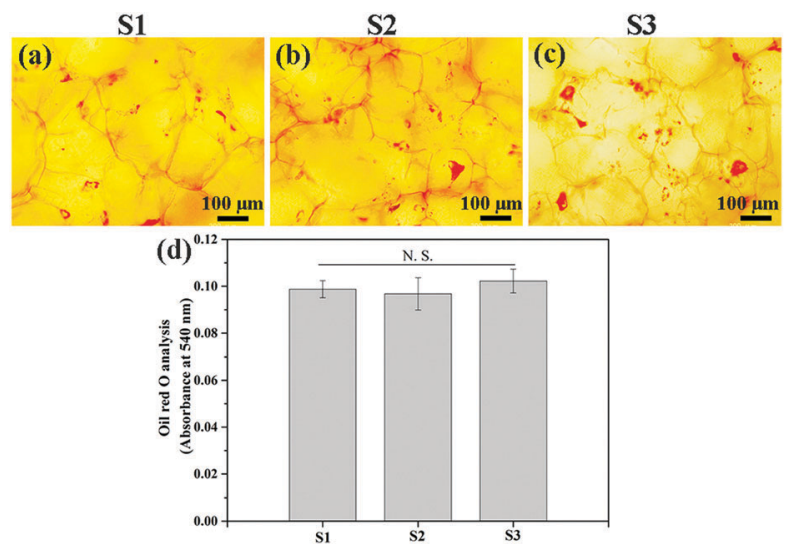

Fig. 9 Oil Red $O$ staining images $(a-c)$ and quantification of extracted Oil Red $O$ dye (d) of cell/scaffold constructs after the hMSCs were cultured in the gelation scaffold (S1 and a), $2.0 \mathrm{mM}$ AuNRs-gelatin scaffold (S2 and b) and $4.0 \mathrm{mM}$ AuNRs-gelatin scaffold ( $\mathrm{S} 3$ and c) for 14 days. The data are presented as mean \pm standard deviation, $n=3$. No significant difference: N.S. indicated that the gelatin scaffold and AuNRs-gelatin composite scaffolds had the same promotive effects on the adipogenic differentiation of hMSCs. The incorporation of AuNRs in the gelatin scaffold had no influence on the promotive effect of the gelatin scaffold for the adipogenic differentiation of hMSCs.

\section{Discussion}

The complete ablation of residual breast tumor cells and the successful reconstruction of tumor-initiated breast defects after surgical resection remain a critical challenge in breast cancer therapy. ${ }^{43}$ To address these issues, bifunctional AuNRs-gelatin composite porous scaffolds were designed and prepared by incorporating photothermal conversion nanoparticles, AuNRs, in a gelatin porous scaffold. AuNR aqueous solutions at different concentrations of AuNRs (2.0 and $4.0 \mathrm{mM}$ ) were used to introduce different amounts of AuNRs into the composite scaffolds. Preprepared ice particulates were used as a porogen material to control the pore structure of the composite scaffolds. The composite scaffolds had the same size of spherical large micropores and small micropores as those of the gelatin scaffold (Fig. 2a-c). The spherical large micropores were well interconnected by the small micropores on the walls of the spherical large micropores. All the gelatin and composite scaffolds had the same pore structures because the same ice particulates were used and the freezing temperature was the same during the preparation process. The size and shape of the spherical large micropores were controlled by the pre-prepared ice particulates because they were the negative replicas of the pre-prepared ice particulates. The small micropores on the walls of the large micropores should be the replica of the new ice crystals that were formed among the pre-prepared ice particulates during the freezing process of the ice particulates-AuNRs-gelatin mixture solution. The porous structure and good interconnectivity of the composite scaffolds were beneficial for cell adhesion and distribution throughout the scaffolds, as shown in Fig. 7.

The synthesized AuNRs had a strong near-infrared light absorption peak at a wavelength of $813 \mathrm{~nm}$ (Fig. 1d), which is skin-penetrative, non-invasive and harmless. ${ }^{44}$ The AuNRsgelatin composite scaffolds exhibited excellent photothermal performance under NIR (805 nm) irradiation (Fig. 3 and Table 2). The photothermal conversion of the composite scaffolds should be due to the photothermal conversion properties of the AuNRs. It has been reported that AuNRs can efficiently convert NIR light into heat. ${ }^{45}$ The photothermal-induced temperature changes of the AuNRs-gelatin scaffolds could be modulated by changing the laser power intensity, irradiation time and incorporated amount of AuNRs.

The excellent photothermal performance of the AuNRsgelatin composite scaffolds was used for the photothermal ablation of breast tumor cells. The in vitro cell culture and in vivo animal experiments showed that the composite scaffolds could effectively kill breast tumor cells (Fig. 4 and 5). The composite scaffolds prepared with different concentrations of AuNRs (2.0 mM and $4.0 \mathrm{mM}$ ) were used for the ablation of tumor cells to examine the effect of AuNR amount. The in vitro cell 


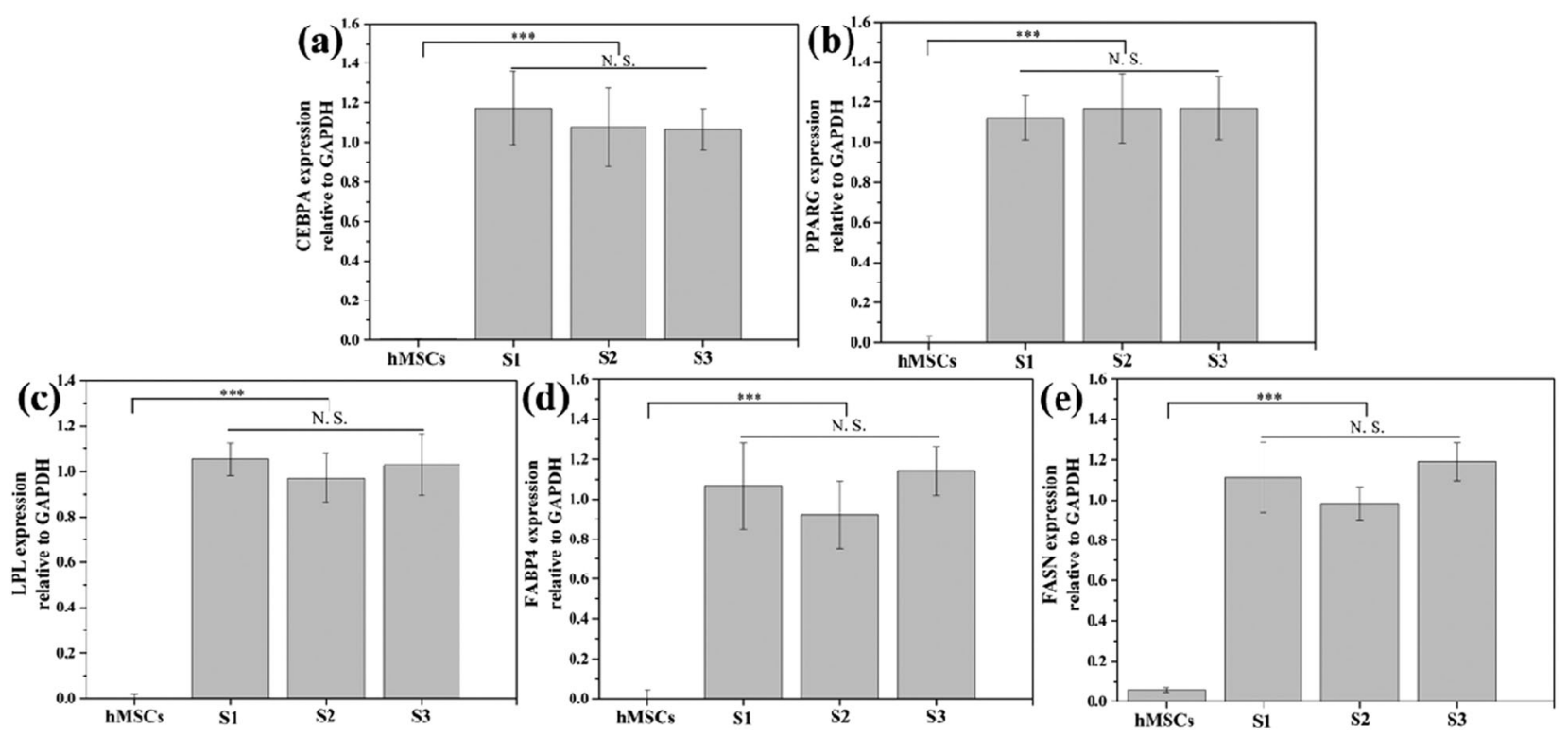

Fig. 10 Expression of genes encoding CEBPA (a), PPARG (b), LPL (c), FABP4 (d) and FASN (e) by hMSCs cultured in the gelatin scaffold (S1), 2.0 mM AuNRs-gelatin scaffold (S2) and $4.0 \mathrm{mM}$ AuNRs-gelatin scaffold (S3) for 14 days. The subcultured hMSCs used for cell seeding were used as the control. Data are presented as mean $\pm \mathrm{SD}, n=3$. No significant difference: N.S.; significant difference: ${ }^{* \star *} p<0.001$.

culture experiments indicated that the two composite scaffolds could effectively kill tumor cells and the killing effect increased with the increased amount of incorporated AuNRs, NIR laser intensity and irradiation time (Fig. 4). The dependence of the tumor cell ablation capacity on the amount of AuNRs, NIR laser intensity and irradiation time should be due to the heating characteristics of the AuNRs incorporated in the composite scaffolds. Because both $2.0 \mathrm{mM}$ and $4.0 \mathrm{mM}$ AuNRs-gelatin scaffolds could kill all the breast tumor cells after NIR laser irradiation at a power intensity of $1.3 \mathrm{~W} \mathrm{~cm}^{-2}$ for 8 minutes and a power intensity of $1.6 \mathrm{~W} \mathrm{~cm}^{-2}$ for 6 minutes, the $2.0 \mathrm{mM}$ AuNRs-gelatin scaffold was used for the in vivo animal experiments.

For the in vivo animal experiments, MDA-MB231-Luc cells were seeded in the scaffolds and then subcutaneously implanted in nude mice. The seeded cells could be thought of as migrated cells from the surrounding tissues and this model was used to confirm the killing effect of the composite scaffolds on the migrated cells. NIR laser irradiation at a power intensity of $1.3 \mathrm{~W} \mathrm{~cm}^{-2}$ for 10 minutes and a power intensity of $1.6 \mathrm{~W} \mathrm{~cm}^{-2}$ for 8 minutes was used to guarantee the killing effect. The whole-body bioluminescence images showed that the luminescence intensity, which indicated living cells, decreased dramatically after the NIR laser irradiation of the $2.0 \mathrm{mM}$ AuNRs-gelatin scaffold (Fig. 5). The results indicated that most of the breast cancer cells were killed by the photothermal ablation of the $2.0 \mathrm{mM}$ AuNRs-gelatin composite scaffolds. Both irradiation conditions at a power intensity of 1.3 for 10 minutes and a power intensity of $1.6 \mathrm{~W} \mathrm{~cm}^{-2}$ for 8 minutes showed dramatic ablation effects. However, the skin tissue near the $2.0 \mathrm{mM}$ AuNRs-gelatin scaffolds was obviously damaged after laser irradiation at a high power intensity (1.6 $\mathrm{W} \mathrm{cm}^{-2}$ for 8 minutes) (Fig. 6). Therefore, achieving effective tumor ablation at a relatively low photothermal temperature is critical toward successful clinical application of PTT. ${ }^{46}$
Besides the efficient photothermal ablation of tumor cells, adipose tissue regeneration is required to realize breast reconstruction to improve the quality of life of patients. The potential of the AuNRs-gelatin composite scaffolds for adipose tissue engineering was confirmed by examining their promotive effect on the adipogenic differentiation of hMSCs. The composite scaffolds not only supported the adhesion and proliferation of hMSCs, as shown in Fig. 7 and 8, but they also enhanced the formation of lipid vacuoles (Fig. 9) and the expression of adipogenic genes (CEBPA, PPARG, LPL, FABP4 and FASN) (Fig. 10). In addition, the composite scaffolds showed the same level of lipid vacuole formation and the same expression level of these genes as the gelatin scaffold, which indicated that the incorporation of AuNRs had no influence on the adipogenic differentiation of hMSCs. Although the in vivo adipogenesis promotion effect of the composite scaffolds will be further confirmed in future, the results in the present study indicated that the AuNRs-gelatin composite scaffolds exhibited a bifunctional property for the simultaneous photothermal ablation of breast tumor and the adipogenic differentiation of hMSCs.

\section{Conclusions}

Bifunctional AuNRs-gelatin porous scaffolds were prepared by introducing AuNRs in the porous structure of gelatin matrices via a freeze-drying method. The pre-prepared ice particulates were used as templates to control the pore structure. The AuNRs-gelatin scaffolds exhibited high photothermal conversion efficiency and excellent photothermal ablation capacity toward breast tumor cells in vitro and in vivo. Furthermore, the composite scaffolds supported adhesion, promoted proliferation and enhanced the adipogenic differentiation of hMSCs. The results 
demonstrated that the AuNRs-gelatin scaffolds could exhibit the simultaneous photothermal ablation of breast tumor cells and regeneration of adipose tissue. The composite scaffolds should be useful for the treatment of breast tumors.

\section{Conflicts of interest}

There are no conflicts to declare.

\section{Acknowledgements}

This work was supported by JSPS KAKENHI Grant Number 18K19947 and 18K19945.

\section{References}

1 H. Li, K. Wang, X. Yang, Y. Zhou, Q. Ping, D. Oupicky and M. Sun, Acta Biomater., 2017, 53, 399-413.

2 D. S. Micalizzi and S. Maheswaran, Nature, 2018, 554, 308-309.

3 R. Ruda, F. Bruno and R. Soffietti, Current treatment options in neurology, 2018, 20, 33.

4 P. Quirke, P. Durdey, M. F. Dixon and N. S. Williams, Lancet, 1986, 2, 996-999.

5 G. R. Stark, Nature, 1986, 324, 407.

6 D. M. Busenhart, J. Erb, G. Rigakos, T. Eliades and S. N. Papageorgiou, Oral Oncol., 2018, 83, 64-72.

7 R. Robinson, L. Marconi, E. MacPepple, O. W. Hakenberg, N. Watkin, Y. Yuan, T. Lam, S. MacLennan, T. E. Adewuyi, A. Coscione, S. S. Minhas, E. M. Comperat and A. Necchi, Eur. Urol., 2018, 74, 76-83.

8 M. Tachi and A. Yamada, Int. J. Clin. Oncol., 2005, 10, 289-297. 9 A. R. K. Sasikala, A. R. Unnithan, R. G. Thomas, S. W. Ko, Y. Y. Jeong, C. H. Park and C. S. Kim, Adv. Funct. Mater., 2018, 28, 1704793.

10 X. Wang, T. Li, H. Ma, D. Zhai, C. Jiang, J. Chang, J. Wang and C. Wu, NPG Asia Mater., 2017, 9, e376.

11 L. Cheng, C. Wang, L. Feng, K. Yang and Z. Liu, Chem. Rev., 2014, 114, 10869-10939.

12 Y. Li, W. Lu, Q. Huang, M. Huang, C. Li and W. Chen, Nanomedicine, 2010, 5, 1161-1171.

13 Y. W. Chen, Y. L. Su, S. H. Hu and S. Y. Chen, Adv. Drug Delivery Rev., 2016, 105, 190-204.

14 D. Jaque, L. Martinez Maestro, B. del Rosal, P. Haro-Gonzalez, A. Benayas, J. L. Plaza, E. Martin Rodriguez and J. Garcia Sole, Nanoscale, 2014, 6, 9494-9530.

15 B. Jang, J. Y. Park, C. H. Tung, I. H. Kim and Y. Choi, ACS Nano, 2011, 5, 1086-1094.

16 C. W. Hsiao, E. Y. Chuang, H. L. Chen, D. Wan, C. Korupalli, Z. X. Liao, Y. L. Chiu, W. T. Chia, K. J. Lin and H. W. Sung, Biomaterials, 2015, 56, 26-35.

17 E. Blanco, H. Shen and M. Ferrari, Nat. Biotechnol., 2015, 33, 941-951.

18 H. Ma, C. Jiang, D. Zhai, Y. Luo, Y. Chen, F. Lv, Z. Yi, Y. Deng, J. Wang, J. Chang and C. Wu, Adv. Funct. Mater., 2016, 26, 1197-1208.
19 J. Zhang, J. Li, S. Chen, N. Kawazoe and G. Chen, J. Mater. Chem. B, 2016, 4, 5664-5672.

20 Y. Zhang, D. Zhai, M. Xu, Q. Yao, J. Chang and C. Wu, J. Mater. Chem. B, 2016, 4, 2874-2886.

21 J. Zhang, J. Li, N. Kawazoe and G. Chen, J. Mater. Chem. B, 2017, 5, 245-253.

22 E. Rossi, J. Guerrero, P. Aprile, A. Tocchio, E. A. Kappos, I. Gerges, C. Lenardi, I. Martin and A. Scherberich, Acta Biomater., 2018, 73, 154-166.

23 E. J. Combellack, Z. M. Jessop, N. Naderi, M. Griffin, T. Dobbs, A. Ibrahim, S. Evans, S. Burnell, S. H. Doak and I. S. Whitaker, Gland surgery, 2016, 5, 227-241.

24 S. Ogino and N. Morimoto, J. Tissue Eng. Regener. Med., 2018, 12, 633-641.

25 L. Hong, I. Peptan, P. Clark and J. J. Mao, Ann. Biomed. Eng., 2005, 33, 511-517.

26 P. H. Maurer, J. Exp. Med., 1957, 8, 125-131.

27 P. H. Maurer, J. Exp. Med., 1954, 100, 515-523.

28 Y. Kimura, T. Inamoto and Y. Tabata, J. Biomater. Sci., Polym. Ed., 2010, 21, 463-476.

29 Y. Kimura, W. Tsuji, H. Yamashiro, M. Toi, T. Inamoto and Y. Tabata, J. Tissue Eng. Regener. Med., 2010, 4, 55-61.

30 Y. Kimura, M. Ozeki, T. Inamoto and Y. Tabata, Biomaterials, 2003, 24, 2513-2521.

31 D. W. Hutmacher, Biomaterials, 2000, 21, 2529-2543.

32 E. Mele, J. Mater. Chem. B, 2016, 4, 4801-4812.

33 H. Zhu, D. Zhai, C. Lin, Y. Zhang, Z. Huan, J. Chang and C. Wu, J. Mater. Chem. B, 2016, 4, 6200-6212.

34 J. J. Yoon and T. G. Park, J. Biomed. Mater. Res., 2001, 55, 401-408.

35 X. Wu, Y. Liu, X. Li, P. Wen, Y. Zhang, Y. Long, X. Wang, Y. Guo, F. Xing and J. Gao, Acta Biomater., 2010, 6, 1167-1177.

36 Q. Zhang, H. Lu, N. Kawazoe and G. Chen, Acta Biomater., 2014, 10, 2005-2013.

37 H. H. Oh, Y. G. Ko, H. Lu, N. Kawazoe and G. Chen, Adv. Mater., 2012, 24, 4311-4316.

38 Y. Xiang, X. Wu, D. Liu, L. Feng, K. Zhang, W. Chu, W. Zhou and S. Xie, J. Phys. Chem. C, 2008, 112, 3203-3208.

39 J. Li, J. E. J. Li, J. Zhang, X. Wang, N. Kawazoe and G. Chen, Nanoscale, 2016, 8, 7992-8007.

40 S. Chen, Q. Zhang, T. Nakamoto, N. Kawazoe and G. Chen, Tissue Eng., Part C, 2016, 22, 189-198.

41 J. Li, Y. Chen, N. Kawazoe and G. Chen, Nano Res., 2018, 11, 1247-1261.

42 Y. Chen, J. Li, N. Kawazoe and G. Chen, J. Mater. Chem. B, 2017, 5, 6801-6810.

43 A. K. Dutra, W. P. Andrade, S. M. Carvalho, F. B. Makdissi, E. K. Yoshimatsu, M. C. Domingues and M. S. Maciel, J. Plast. Reconstr. Aesthetic Surg., 2012, 65, 187-194.

44 X. Wu, G. Chen, J. Shen, Z. Li, Y. Zhang and G. Han, Bioconjugate Chem., 2015, 26, 166-175.

45 M. A. Mackey, M. R. Ali, L. A. Austin, R. D. Near and M. A. El-Sayed, J. Phys. Chem. B, 2014, 118, 1319-1326.

46 X. Wang, F. Lv, T. Li, Y. Han, Z. Yi, M. Liu and J. Chang, ACS Nano, 2017, 11, 11337-11349. 